\title{
Parents' interpretations of children's respiratory symptoms on video
}

\author{
R S Cane, S A McKenzie
}

\begin{abstract}
Aims-To investigate how parents report children's respiratory sounds on video compared to a clinical "gold standard". Methods-Five clinicians agreed on 10 video clips of children with audible breathing. These responses were the "gold standard". The clips were shown to parents of children: (a) with asthma/wheeze; (b) with other respiratory complaints; (c) without respiratory complaints. Parents were asked what they called the sounds, where they originated, and whether their own child made similar sounds.

Results-A total of 190 parents took part. The "correct" labelling of wheeze was 59\% $(95 \%$ confidence interval 52 to $66 \%$ ) and $47 \%$ (95\% confidence interval 40 to $54 \%$ ) for other sounds (stridor, snoring, stertor). Parents were better at locating both sounds than labelling. There were no differences between subject groups. There were more false positive responses to labelling and locating other sounds than for wheeze $(27 \% v 8 \%$ and $33 \% v 10 \%)$.

Conclusion-Parents locate sounds better than describing them. At least $30 \%$ of all parents use other words for wheeze and $30 \%$ labelled other sounds as "wheeze". This could have important clinical implications.

(Arch Dis Child 2001;84:31-34)
\end{abstract}

Keywords: parents' reports; wheeze; video

A report on medical education in $1968^{1}$ placed the term "taking the history" in inverted commas. This was to make students aware that vocabulary and cultural attitudes may "impede or distort" communication ${ }^{2}$ for both interviewer and interviewee. ${ }^{34}$

Diagnosing childhood asthma is dependent on parental symptom reporting. There are a number of difficulties: some parents confuse respiratory sounds, ${ }^{5}$ night time symptoms are difficult to quantify, ${ }^{6}$ recollection of symptoms may change, ${ }^{7}$ parents' and children's reports of symptom frequency may be discordant, ${ }^{8}$ clinicians' and parents' words for symptoms ${ }^{9}$ and definitions ${ }^{10}$ may differ and, lastly, "wheeze" does not translate into some languages. ${ }^{11}$

To address problems of history taking, we wanted to find out whether breathing sounds of children shown on a video could aid diagnosis. The aims were:

(1) To compare responses to children's respiratory sounds observed on video in the following groups:

- parents and clinicians
- parents of children with and without respiratory symptoms

- parents with and without English as a first language.

(2) To investigate whether videos help parents identify the respiratory sounds of their own children.

\section{Methods}

COMPILATION OF VIDEO CLIPS

Children aged less than 7 years with audible breathing sounds, attending children's hospital services were filmed. A hand held Panasonic Movie Camera was used with an additional clip microphone (Monacor ECM-3005). Children were filmed lying, sitting, or standing in the hospital wards or side rooms. Clips with minimal distracting material in the background, showing clear signs, with good acoustic quality were selected from 55 filmed. Fifteen clips were edited for clinicians to view. Parents' written consent for filming was obtained in all cases.

Clinicians at different levels of training were asked independently to label and locate the breathing sounds on the videos. Only clips showing single clinical features were used. Agreement was reached between five clinicians on 10 of 15 clips. The breathing sounds in the clips were classified as either "wheeze" or "sounds other than wheeze" (stridor, snoring, stertor-referred to here as "other sounds"). Locations were classified as "chest", "nose", and "throat" for each type of sound. These were considered the "gold standard" with which parents' responses were compared.

Three tapes were compiled by age using the 10 agreed clips: <1 year tape showed two infants with wheeze and two with other sounds; 1-4 year tape showed one child with wheeze and two with other sounds; 4-7 year tape showed two children with wheeze and one with another sound. Each clip lasted 80-100 seconds. There are different numbers and combinations of sounds for each age group because it was not possible to get an equal number for each sound for each combination of age, sex, and ethnic origin for which there was clinician agreement. The tapes showed children of each sex and were ethnically representative of the population of east London.

\section{SUBJECTS}

Subjects were 190 parents with child patients aged less than 8 years. Videos were shown in quiet areas usually using a 14 inch portable Philips TV Combi, the same monitor that was used to show clinicians these clips.

Subject groups were parents of children: 
Table 1 Examples of how parents' responses were coded for wheeze clips

\begin{tabular}{lll}
\hline Grouping & Examples for label & Examples for location \\
\hline Correct & Wheeze; asthma; whistling & Chest \\
Vague & Rapid, shallow breaths; chesty & Chest + nose \\
Don't know & Don't know; left blank & - \\
Wrong & Normal breathing; snoring & Nose \\
\hline
\end{tabular}

Table 2 Examples of how parents' responses were coded for other sounds

\begin{tabular}{llll}
\hline \multirow{2}{*}{$\begin{array}{l}\text { Parents' response } \\
\text { grouping }\end{array}$} & Clinicians' responses & & \\
\cline { 2 - 4 } & Stridor & Snoring & Stertor \\
\hline Correct & Sucking in and out & Snuffle; gurgle; snoring & Congested cold \\
Vague & Snuffly & Heavy breathing & Heavy breathing \\
Don't know & Don't know & - & - \\
Wrong & Wheeze & - & -
\end{tabular}

For the location question, "throat" would be a "correct" response for each of these three sounds (and/or "nose" also for the snoring and stertor clips) and "chest" a "wrong" response.

Table 3 Example of how final results were derived. Responses from the asthmatic/wheezy group (group A) in correctly describing the sounds in the wheeze and other sounds clips

\begin{tabular}{|c|c|c|c|c|c|c|c|c|c|}
\hline \multirow{2}{*}{$\begin{array}{l}\text { Age } \\
\text { group }\end{array}$} & \multirow[b]{2}{*}{ No. * } & \multicolumn{4}{|c|}{ Wheeze clips } & \multicolumn{4}{|c|}{ Other sounds } \\
\hline & & $n=1 / 1$ & $n=1 / 2$ & $n=2 / 2$ & Total & $n=1 / 1$ & $n=1 / 2$ & $n=2 / 2$ & Total \\
\hline$<1$ & 3 & - & 1 & 1 & 2 & - & 0 & 0 & 0 \\
\hline $1-4$ & 39 & 17 & - & - & 17 & - & 9 & 4 & 13 \\
\hline $4-7$ & 40 & - & 20 & 13 & 33 & 20 & - & - & 20 \\
\hline All & 82 & & & & 52 & & & & 33 \\
\hline
\end{tabular}

(A) who had been diagnosed with asthma or had been observed by doctors to wheeze

(B) who had other respiratory symptoms

(C) who had no respiratory problems or history of asthma (controls).

Parents watched the video tape of children in the same age group as their own child. An accompanying questionnaire asked two questions for each clip: "what do you call the sounds you hear this child making?" (referred to as as the "label question") and "where do you think the sounds are coming from-the nose, throat, or chest?" (the "location question"). Responses were open ended so parents used their own words. Finally, parents were asked if any clip resembled the breathing sounds their own child made. Responses were checked against each child's medical record. Responses were either recorded in writing by the parent or a trained interviewer. The only prompts used were "if your child was making a sound like this think how you would describe it to someone else" and "if you're not sure, you can leave it blank".

Parents were asked their first language. The questionnaire was available in English, Urdu, Bengali (Sylheti), and Turkish, the main local languages and supplied by hospital community health advocates. Parents were given the opportunity to watch the video twice, to have the sound volume altered, and to ask questions at the end. The project was approved by the local ethics committee.

DATA ANALYSIS

Comparison of responses to respiratory sounds between groups

As parents used their own words in responding to the clips, categories for these answers were devised. These were concordant ("correct"), or discordant ("vague", “don't know", and "wrong") in comparison to the clinicians' gold standard for each wheeze and other sound clips. To help distinguish between the "vague" and "correct" categories for the label question, a list of 24 parents' responses were given to seven clinicians in the paediatric respiratory team. They were asked whether these labels corresponded to wheeze, other sounds, or were ambiguous. Tables 1 and 2 show examples of parents' responses in each of the four categories to the label and location questions for "wheeze" and "other sounds" clips (the latter group has been subdivided according to the type of sound). Inter-rater reliability for assigning categories to parents' responses was higher than $95 \%$ (anybody classifying these responses would assign the same categories most of the time). Result tables show only "wrong" responses (as false positives) and "correct" responses.

As parents watched the video clips of children the same age as their own child and different age groups had different numbers of "wheeze" and "other sounds" clips, all analyses are made on the basis of at least one correct or wrong response per parent. Table 3 shows an example of how correct results for group A for the label question for each type of clip were derived. Results are reported in percentages with $95 \%$ confidence intervals (CI) and comparisons made by $\chi^{2}$ testing.

Parents' comparison of video sounds with sounds made by their own child

If parents indicated that a child on the video sounded like their own child, this was compared to clinicians' impressions recorded in the child's medical record. Comparisons were scored as "match" or "mismatch". Any indication of similarity was then related to the parents' initial labelling of the sound to investigate whether the video helped parents to communicate their child's symptoms.

\section{Results}

Over a period of 12 months, 294 adults were asked to watch the video; 86 declined $(75 \%$ female and $55 \%$ of south Asian origin or descent) and 18 were not included (communication too problematic).

Of the 190 in the study, 82 belonged to group A, 56 to group B, and 52 to group C. Overall, $74 \%$ of respondents were mothers, $11 \%$ fathers, $10 \%$ of responses were by both parents, and $5 \%$ were by relatives of the patient. Fifty seven per cent were British, 25\% of south Asian origin or descent, 9\% African, $5 \%$ Turkish, 3\% European, and 1\% other.

COMPARISON OF RESPONSES BETWEEN GROUPS Parents and gold standard

Table 4 shows the results for an overall comparison between responses from the 190 parents and the gold standard. Parents were more likely to correctly label wheeze than other sounds, but better able to locate other sounds than wheeze.

Wrong labelling of sounds, for example calling wheeze "snoring" or calling snoring 
Table 4 Number (percentage, 95\% CI) of all parents $(n=190)$ who correctly and wrongly labelled and located both types of sounds at least once

\begin{tabular}{lllll}
\hline Question & Correct & p value & Wrong (false +$)$ & p value \\
\hline $\begin{array}{l}\text { Label } \\
\text { Wheeze }\end{array}$ & $113(59 \%, 52-66 \%)$ & 0.02 & $16(8 \%, 4-12 \%)$ & $<0.001$ \\
$\begin{array}{l}\text { Other } \\
\text { Locate }\end{array}$ & $90(47 \%, 40-54 \%)$ & & $51(27 \%, 21-33 \%)$ & \\
$\quad \begin{array}{l}\text { Wheeze } \\
\text { Other }\end{array}$ & $132(69 \%, 62-76 \%)$ & 0.01 & $19(10 \%, 6-14 \%)$ & $<0.001$ \\
\hline
\end{tabular}

Correct label versus location for wheeze: $\mathrm{p}=0.05$.

Correct label versus location for other sounds: $\mathrm{p}<0.001$

"wheeze", was more likely to occur with the other sounds-that is, the other sounds were mislabelled wheeze or asthma. Of 51 parents who gave a false positive response to other sounds, 21 used the word "wheeze", seven "asthma", three "chesty", and 20 gave combinations of these and other words. Parents were also more likely to wrongly locate other sounds; of 62 wrong responses, 53 said they were from the chest.

Parents of children with wheeze, other respiratory symptoms, and controls

Table 5 shows results by subject group for both label and location questions to both types of sounds. There was no significant difference between groups for correct/wrong labelling/ locating of sounds. The maximum $95 \%$ confidence limit for labelling wheeze correctly was less than $75 \%$.

Responses of parents by their first language Table 6 shows correct and wrong results for parents' responses by language group. English was a first language for $112 / 190$ (59\%). The remainder included those who spoke fluent English and those who used translations (six in Turkish and four in Bengali). Where translations were used, responses were translated into English.

Parents with English as a first language were more accurate at labelling each sound than other parents (all sounds combined: 63\% v $40 \%, \mathrm{p}<0.001)$. Responses were more similar between groups for the location question (all sounds combined: $80 \% v 69 \%, \mathrm{p}=0.01)$.
PARENTS' IDENTIFICATIONS OF VIDEO CLIP TO OWN CHILD

Of 82 parents in group A, 17 (20\% CI: $11-29)$ did not recognise wheeze although their child had previously been observed to wheeze or had been diagnosed with asthma. Sixty five $(79 \%$ CI: 70-88) identified a clip as similar to their child, of which 14 indicated both types of sounds and seven "other sounds" alone. Of the 44 who identified the wheeze clip, 29 (66\%) had previously labelled the sound correctly and $15(34 \%)$ had not.

\section{Discussion}

The use of video in research is beneficial. It is relatively free from biases caused by language, culture, literacy, or interviewing technique, ${ }^{12}$ and may allow more valid and repeatable recognition of symptoms. ${ }^{13}$ It has been used to validate written questionnaires, ${ }^{12}$ as an epidemiology tool with older children, ${ }^{14}$ to measure night time symptoms, ${ }^{15}$ as part of an educational programme with asthmatics, ${ }^{16}$ and as "video diaries" showing clinicians the life context for asthmatics managing their symptoms. ${ }^{17}$ To the best of our knowledge, it has not been used to assess parents' interpretation of children's respiratory symptoms.

The recording of good quality videos proved difficult and even then agreement between clinicians was unanimous for only 10 of 15 clips. We believe the final selection showed a clear distinction between the sounds. In real life this may not be so evident. Our subjects were parents of children attending children's services in a general hospital. Two of the three subject groups were parents whose children were known to have asthma or other respiratory problems. It seems reasonable to assume that these groups would be more concordant with clinicians' interpretations of the video clips than parents of children with no history of respiratory symptoms. However, this was not the case. For this study we deliberately used video clips of children with single auditory sounds. In real life this is often not the case. Upper airway noise, for example related to nasal discharge, may accompany wheeze. Fourteen parents of

Table 5 Number (percentage, 95\% CI) of parents who correctly and wrongly labelled and located both types of sounds at least once by subject group

\begin{tabular}{|c|c|c|c|c|c|c|}
\hline \multirow[b]{2}{*}{ Question } & \multicolumn{2}{|l|}{ Group $A(n=82)$} & \multicolumn{2}{|l|}{ Group B $(n=56)$} & \multicolumn{2}{|l|}{ Group $C(n=52)$} \\
\hline & Correct & Wrong & Correct & Wrong & Correct & Wrong \\
\hline \multicolumn{7}{|l|}{ Label } \\
\hline Wheeze & $52(63 \%, 52-74 \%)$ & $6(7 \%, 1-13 \%)$ & $31(55 \%, 42-68 \%)$ & $7(13 \%, 4-22 \%)$ & $30(58 \%, 44-72 \%)$ & $3(6 \%, 0-12 \%)$ \\
\hline Other & $33(40 \%, 29-51 \%)$ & $21(26 \%, 16-36 \%)$ & $28(50 \%, 37-63 \%)$ & $16(29 \%, 17-41 \%)$ & $29(56 \%, 42-70 \%)$ & $14(27 \%, 15-39 \%)$ \\
\hline \multicolumn{7}{|l|}{ Locate } \\
\hline Wheeze & $60(73 \%, 63-83 \%)$ & $5(6 \%, 1-11 \%)$ & $37(66 \%, 53-79 \%)$ & $10(18 \%, 8-28 \%)$ & $35(67 \%, 54-80 \%)$ & $4(8 \%, 0-16 \%)$ \\
\hline Other & $64(78 \%, 69-87 \%)$ & $23(28 \%, 18-38 \%)$ & $46(82 \%, 72-92 \%)$ & $20(36 \%, 23-49 \%)$ & $44(85 \%, 75-95 \%)$ & $19(37 \%, 24-50 \%)$ \\
\hline
\end{tabular}

Table 6 Number (percentage, 95\% CI) of parents who correctly and wrongly labelled and located both types of sounds at least once by language group

\begin{tabular}{|c|c|c|c|c|c|c|}
\hline \multirow[b]{2}{*}{ Question } & \multicolumn{3}{|l|}{ Correct responses } & \multicolumn{3}{|l|}{ Wrong responses } \\
\hline & English $(n=112)$ & Other language $(n=78)$ & $p$ value & English $(n=112)$ & Other language $(n=78)$ & $p$ value \\
\hline \multicolumn{7}{|l|}{ Label } \\
\hline Wheeze & $75(76 \%, 58-76 \%)$ & $38(49 \%, 38-60 \%)$ & 0.02 & $10(9 \%, 4-14 \%)$ & $6(8 \%, 2-14 \%)$ & NS \\
\hline Other & $66(59 \%, 50-68 \%)$ & $24(31 \%, 21-41 \%)$ & $<0.001$ & $26(23 \%, 15-31 \%)$ & $25(32 \%, 21-43 \%)$ & NS \\
\hline \multicolumn{7}{|l|}{ Locate } \\
\hline Wheeze & $81(72 \%, 64-80 \%)$ & $52(65 \%, 55-75 \%)$ & NS & $11(9 \%, 4-14 \%)$ & $8(10 \%, 3-17 \%)$ & NS \\
\hline Other & $99(88 \%, 82-94 \%)$ & $55(72 \%, 62-82 \%)$ & 0.004 & $32(29 \%, 20-38 \%)$ & $30(38 \%, 27-49 \%)$ & NS \\
\hline
\end{tabular}


wheezy or asthmatic children indicated that their child had both types of sounds. However, as in "real life" we did allow parents to say they "don't know" to our questions because we felt that demanding a response could skew the data. Often parents responding to epidemiology surveys are not given this option. ${ }^{10}$ It is also important to acknowledge that our parents watched a sequence of clips which may have had a "carry over" effect on their responses (for example, accumulating comparisons).

As parents were more concordant with clinicians in locating sounds, especially "other sounds", this suggests that questions about location may be more helpful than a description, especially where English is not the first language. In 1990-91 the risk of underdiagnosis and undertreatment of asthma was higher in children from minority ethnic groups. ${ }^{18}$ Rather than analysing responses with respect to ethnicity, we used language instead because some words like "wheeze" do not have exact equivalence in some languages. There was a disproportionate number of Asian parents who refused to take part, possibly because of initial communication difficulties.

By asking parents whether any clip resembled their own child's symptoms, we could assess the use of audiovisual prompts. For example, a mother may say that her own child was "just like this one" on the video and the medical files confirm this, but in the earlier question when asked to label that clip, she gave a "wrong" response. This was the case in a third of parents with asthmatic or wheezy children who said their child resembled a wheezy child. This suggests that audiovisual aid in consultations could be useful for some parents. However, it was surprising that a fifth of parents of children with asthma or wheeze did not perceive any similarity with our video clips. As perception of similarity is highly subjective, it may be that some parents were more discriminating than others. However, as diagnosis of childhood asthma relies on parentally reported sounds, can we assume a "doctor diagnosis" is correct? ${ }^{10}$ As already mentioned, real life sounds may not be as clear cut as those we chose.

Parental reports of symptom history are very hard to verify. This study has shown that asking parents to locate children's breathing sounds is more concordant with clinicians understanding than a description of them, particularly for those whose first language is not English. At least $30 \%$ of parents, whether they have wheezy children or not, use other words for wheeze and about the same proportion also falsely label other sounds as wheeze $(100 \%$ minus the upper confidence limit). An audiovisual presentation from which to select symptom similarity may be more helpful in consultations for some parents than a verbal description. Such a system needs evaluation in the community.

We thank parents for their participation, medical staff for their help, and Allan Hackshaw at the Wolfson Institute for advice with analysis. RS Cane was supported by the NHS Executive North Thames-Inner City Health Research and Development Group (Perceived respiratory symptoms in children in East London). This paper was presented at the Royal College of Paediatrics and Child Health Spring meeting, 12 April 2000.

1 Todd Report. Report on the Royal Commission of Medical Education 1965-68. London: HMSO, 1968.

2 Armstrong D. Political anatomy of the body; medical knowledge in Britain in the 20th century. Cambridge: Cambridge Uniin Britain in the 20th cen

3 Helman C. Culture, health and illness, 3rd edn. London: Butterworth Heinmann, 1994:106.

4 Lagerlov P, Leseth A, Matheson I. The doctor-patient relationship and the management of asthma. Soc Sci Med 1998;47:85-91

5 Lee DA, Winslow NR, Speight ANP, Hey EN. Prevalence and spectrum of asthma in childhood. BMf 1983;286: $1256-8$

6 Fuller P, Picciotto A, Davis M, McKenzie SA. Cough and sleep in inner-city children. Eur Respir F 1998;12:426-31.

7 Peat JK, Salome CM, Toelle BG, Bauman A, Woolcock AJ. Reliability of a respiratory history questionnaire and effect of mode of administration on classification of asthma in children. Chest 1992;102:153-7.

8 Wong TW, Yu TS, Lui JL Y, Wong SL. Agreement on responses to respiratory illness questionnaire. Arch Dis responses to respiratory
Child $1998 ; 78: 379-80$

9 Ostergaard MS. Childhood asthma: parents' perspective-a qualitative interview study. Fam Pract 1998;15:153-7.

10 Cane RS, Ranganathan SC, McKenzie SA. What do parents of wheezy children understand by "wheeze"? Arch Dis Child 2000;82:327-32.

1 Pararajasingam CD, Sittampalam L, Damani P, Pattemore PK, Holgate ST. Comparison of the prevalence of asthma among Asian and European children in Southampton. Thorax 1992;47:529-32.

12 Shaw RA, Crane J, Pearce N, et al. Comparison of a video questionnaire with the IUATLD written questionnaire form measuring asthma prevalence. Clin Exp Allergy 1992; 22:561-8.

13 Beasley R, Lai CKW, Crane J, Pearce N. The video questionnaire: one approach to the identification of the asthmatic phenotype. Clin Exp Allergy 1998;28(suppl 1):812.

14 Pearce N, Weiland S, Keil U, et al. Self-reported prevalence of asthma symptoms in children in Australia, England, using the ISAAC protocol. Eur Respir f 1993;6:1455-61.

15 Davis M, Fuller P, Picciotto A, McKenzie SA. Persistent cough in children: a randomised trial of high-dose inhaled corticosteroids. Arch Dis Child 1999;81:38-44.

6 Chmelik F, Doughty A. Objective measurements of compliance in asthma treatment. Ann Allergy 1999;73:527-32.

17 Rich M, Lamola S, Amory C, Schneider L. Asthma in life context: video intervention/prevention assessment (VIA). Pediatrics 2000;105:469-77.

18 Duran-Tauleria E, Rona RJ, Chinn S, Burney P. Influence of ethnic group on asthma treatment in children in 1990-1: national cross sectional study. BMF 1996;313:148-52. 